\title{
Primary debulking surgery versus primary neoadjuvant chemotherapy for high grade advanced stage ovarian cancer: comparison of survivals
}

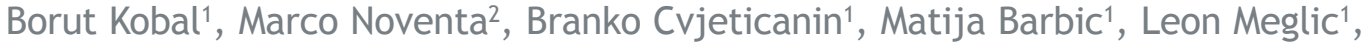 \\ Marusa Herzog ${ }^{1}$, Giulia Bordi ${ }^{3}$, Amerigo Vitagliano², Carlo Saccardi², Erik Skof ${ }^{4}$ \\ ${ }^{1}$ Division of Gynaecology and Obstetrics, University Medical Centre Ljubljana, Ljubljana, Slovenia \\ ${ }^{2}$ Department of Woman and Child Health, University of Padua, Padua, Italy \\ ${ }^{3}$ Department of Gynecology and Obstetrics, University of Insubria, Varese, Italy \\ ${ }^{4}$ Department of Medical Oncology, Institute of Oncology Ljubljana, Ljubljana, Slovenia
}

Radiol Oncol 2018; 52(3): 307-319

Received 28 March 2018

Accepted 23 July 2018

Correspondence to: Assist. Prof. Erik Škof, M.D., Ph.D., Institute of Oncology Ljubljana, Zaloška 2, SI-1000 Ljubljana, Slovenia. Phone: +386 15879 282; Fax: +386 15879 305; E-mail: eskof@onko-i.si

Disclosure: No potential conflicts of interest were disclosed.

Background. The aim of the study was to analyze the overall survival (OS) and progression free survival (PFS) of patients with high grade and advanced stage epithelial ovarian cancer (EOC) with at least 60 months of follow-up treated in a single gynecologic oncology institute. We compared primary debulking surgery (PDS) versus neoadjuvant chemotherapy plus interval debulking surgery (NACT + IDS) stratifying data based on residual disease with the intent to identify the rationale for therapeutic option decision and the role of laparoscopic evaluation of resectability for that intention.

Patients and methods. This is observational retrospective study on consecutive patients with diagnosis of high grade and International Federation of Gynecology and Obstetrics (FIGO) stage III/IV EOC referred to our center between January 2008 and May 2012. We selected only patients with a follow-up of at least 60 months. Primary endpoint was to compare PDS versus NACT + IDS in term of progression free survival (PFS) and overall survival (OS). Secondary endpoints were PFS and OS stratifying data according to residual disease after surgery in patients receiving PDS versus NACT + IDS. Finally, through Cox hazards models, we tested the prognostic value of different variables (patient age at diagnosis, residual disease after debulking, American Society of Anesthesiologists (ASA) stage, number of adjuvantchemotherapy cycles) for predicting OS.

Results. A total number of 157 patients were included in data analysis. Comparing PDS arm (108 patients) and NACT + IDS arm (49 patients) we found no significant differences in term of OS (41.3 versus 34.5 months, respectively) and PFS (17.3 versus 18.3 months, respectively). According to residual disease we found no significant differences in term of OS between NACT + IDS patients with residual disease $=0$ and PDS patients with residual disease $=0$ or residual disease $=1$, as well as no significant differences in PFS were found comparing NACT + IDS patients with residual disease $=0$ and PDS patients with residual disease = 0; contrarily, median PFS resulted significantly lower in PDS patients receiving optimal debulking (residual disease $=1$ ) in comparison to NACT + IDS patients receiving complete debulking (residual disease $=0$ ). PDS arm was affected by a significant higher rate of severe post-operative complications (grade 3 and 4). Diagnostic laparoscopy before surgery was significantly associated with complete debulking.

Conclusions. We confirm previous findings concerning the non-superiority of NACT + IDS compared to PDS for the treatment of EOC, even if NACT + IDS treatment was associated with significant lower rate of post-operative complications. On the other hand, selecting patients for NACT + IDS, based on laparoscopic evaluation of resectabilty prolongs the PFS and does not worse the OS compared to the patients not completely debulked with PDS.

Key words: epithelial ovarian cancer; advanced stage; primary debulking surgery; interval debulking surgery; neoadjuvant chemotherapy; adjuvant chemotherapy; overall survival; progression free survival 


\section{Introduction}

Epithelial ovarian cancer (EOC) is the major cause of gynecological cancer-related mortality in developed countries, with annual incidence of more than 200,000 new cases and responsible of 150,000 deaths worldwide. ${ }^{1}$ Due to its subtle symptomatology and the lack of specific screening methods, about $70 \%$ of EOCs are diagnosed in advanced stage, specifically International Federation of Gynecology and Obstetrics (FIGO) stage III and IV. ${ }^{2}$

Except for patients not eligible for surgery due to severe comorbidities or extensive tumor spread, the standard treatment for advanced stage EOC is primary debulking surgery (PDS), with the goal of optimal cytoreduction followed by adjuvant chemotherapy with paclitaxel plus platinum based agents. ${ }^{2,3}$ Survival in patients affected by EOC is strongly related to the residual disease after surgical treatment. ${ }^{2}$ Patients without macroscopic residual tumor (complete debulking) showed a better survival than patients with minimal residual disease $<1 \mathrm{~cm}$ (optimal debulking) and patients with residual disease $>1 \mathrm{~cm}$ (suboptimal debulking). ${ }^{4}$ The possibility to attain complete cytoreduction depends on several factors like the spread of the disease, the molecular features of the tumor, its microenvironment and the skill of gynecologic oncology surgeon. 5,6

Current evidence suggest that PDS should only be attempted if at least tumor resection to less than $1 \mathrm{~cm}$ seems to be achievable. ${ }^{2}$ This concept leads to an unsolved key problem in EOC care, due to lack of worldwide accepted pre-operative strategies able to predict the chances of successful primary debulking. ${ }^{7}$ Great progresses have been made concerning the role of pre-operative laparoscopy in evaluating the feasibility of a successful PDS; at this regard recent prospective studies demonstrated a good accuracy of laparoscopic score in predicting residual disease after PDS. ${ }^{7}$ However, randomized trials are mandatory to confirm these encouraging results.

For patients in whom a complete cytoreduction during primary surgery is not expected, neoadjuvant chemotherapy (NACT) followed by interval debulking surgery (IDS) is considered the most appropriate therapeutic option. ${ }^{2,8}$ Recent studies demonstrated that such strategy allows higher rate of residual disease $=0$ in comparison to primary surgery ${ }^{9-11}$ Consequently, an approach based on NACT + IDS as first line treatment in all patients suffering by advanced stage EOC has been recently proposed and two randomized controlled studies have been published in order to compare survivals of PDS versus NACT + IDS strategy. ${ }^{12,13}$ Both EORTC and the most recent CHORUS trial showed no differences in overall survival (OS) and progression free survival (PFS) in the two treatments arms. ${ }^{12,13}$ However, concerns about the degree of evidence from these two studies have been raised, especially due to possible bias related with poor surgical radicality, low median operating time and the poor OS reported in entire study population. ${ }^{14}$ Due to these drawbacks, and considering that other recent prospective and retrospective series reached opposite conclusions in favor to PDS approach, a definitive answer on the role of NACT + IDS in the treatment of advanced stage EOC has not been given yet. ${ }^{9-11,15,16}$

Another fundamental aspect in choosing the two treatments option regards the quality of life of patients. Poor evidence is available concerning QoL (Quality of Life) after PDS or NACT + IDS, mainly supporting higher rate of aggressive surgery and surgery-related complications in patients underwent PDS treatment. 9,10,12,17,18 However, such observations need further clinical confirmations.

Starting from such uncertainties about the optimal primary treatment of advanced stage EOC, we analyzed the OS and PFS of patients with at least 60 months of follow-up in a single gynecologic oncology institute. We compared PDS versus NACT + IDS stratifying data based on residual disease after surgery with the intent to identify the rationale for therapeutic option decision and the role of laparoscopic evaluation of resectability for that intention.

\section{Patients and methods}

\section{Study design}

We conducted an observational retrospective study on patients with diagnosis of high grade and advanced stage (FIGO stage III or IV) EOC that underwent primary-debulking surgery (PDS) or neoadjuvant chemotherapy (NACT) plus interval debulking surgery (IDS) at our institution (Division of Gynecology and Obstetrics, University Medical Centre Ljubljana, Slovenia) from January 2008 to May 2012. The institutional review board approved this retrospective analysis (IRB: 178/05/09).

\section{Inclusion and exclusion criteria}

We included all newly diagnosed patients who referred at our institution with stage III or IV disease according to 1988 FIGO staging criteria. ${ }^{2}$ We 
considered as inclusion criteria: diagnosis of high grade epithelial ovarian cancer with FIGO stage III or IV, all histological type, patients treated by PDS or NACT + IDS, follow-up with at least 60 months duration, absence of concomitant malignant neoplasms. We excluded all patients that underwent PDS at an outside facility, FIGO stage I-II, nonepithelial histologic type and low grade.

\section{Data collection}

Patients were identified through our institution computer database initiated to collect surgical information at point of care. For each patient the investigators (G.B and M.N) reviewed the electronic hospital records and pathology reports to determine study eligibility, patients general features, FIGO stage, tumor grading and histologic type. Vital status was determined by analysis of electronic chart; in case of missing information, investigators contacted directly the patient or family by telephone or email to complete the data collection.

We collected data about patients' age, body mass index (BMI), menopausal status, parity, pre-operative CA-125, documented comorbidities, prior surgeries, number of neoadjuvant or adjuvant chemotherapy cycles, type of chemotherapy, response after NACT, blood loss at surgery and need for transfusions, intraoperative complications (PDS or IDS), post-operative complications (PDS or IDS) based on Clavien Dindo classification system, hospitalization length and residual disease after surgery. ${ }^{19}$

\section{Patients and treatments}

Patients included in the study were subsequently divided in two groups for comparison estimates: (1) PDS group included patients that underwent primary debulking surgery followed by adjuvant chemotherapy; (2) NACT + IDS group included patients that underwent neoadjuvant chemotherapy plus interval debulking surgery followed by adjuvant chemotherapy.

All patients in PDS group underwent PDS with the intent to perform a debulking procedure. A Multidisciplinary Group (including a panel of experts in gynecologic oncology, medical oncology, radiology and pathology) established case by case all treatments decisions through consensus. The decision was based on clinical features of each patient (extent of disease, co-morbidity, performance status), on results of imaging techniques (transvaginal/trans-abdominal ultrasonography, computerized tomography, magnetic resonance imag- ing) and for the great majority of cases was based on laparoscopic direct visualization of pelvic and abdominal cavity with the purpose of evaluation for resectability. When the possibility to perform an optimal surgical cytoreduction (residual disease $\leq 1 \mathrm{~cm}$ ) was considered low, patients were deemed eligible for NACT.

At that time, the selection of patients for PDS or NACT was not based on a formal laparoscopic scoring system but on gynecologic oncologist surgeons expertise. However, a patient was considered candidate for NACT in case of wide spread of the disease in the abdominal and pelvic cavity (unresectable massive peritoneal involvement, widespread infiltrating carcinomatosis of diaphragm, mesenteral retraction, miliary carcinomatosis of the bowel, liver and stomach metastases). Chemotherapy in the adjuvant and neoadjuvant setting included platinum and taxane or doxorubicin regimes according to the standard treatment protocols for the time period. Debulking surgery involved hysterectomy, bilateral oophorectomy, complete omentectomy, selective lymphadenectomy plus if necessary bowel or recto-sigmoid resection, and radical upper abdominal procedures (like diaphragm resection, splenectomy, distal pancreatectomy, and liver resection) to achieve optimal cytoreduction.

The goal at that time period was to obtain at least an optimal $(\leq 1 \mathrm{~cm}$ residual disease - residual disease $=1$ ) or complete cytoreduction (residual disease $=0$ ). Patients were considered suboptimally debulked if they had any residual disease larger than $1 \mathrm{~cm}$ in greatest dimension after surgery (residual disease $=2$ ).

\section{Endpoints of the study}

The primary endpoint in this analysis was to compare the two treatments arms in term of progression free survival (PFS) and overall survival (OS).

The secondary endpoint was to compare the progression free survival (PFS) and overall survival (OS) in patients that underwent NACT + IDS with complete cytoreduction (residual disease $=0$ ) versus patients that underwent PDS with optimal (residual disease $=1$ ) or complete (residual disease $=0)$ cytoreduction.

Tertiary endpoint was to test (using Cox proportional hazards models) the following prognostic factors for OR: type of treatment (PDS versus $\mathrm{NACT}+$ IDS), patient age at diagnosis ( $<60$ versus $\geq 60$ years), residual disease after surgical debulking (residual disease $=0$ versus residual disease $=$ 
TABLE 1. Patient characteristics by treatment arm: primary debulking surgery (PDS) $(N=108)$ versus neoadjuvant chemotherapy (NACT) $(N=49)$

\begin{tabular}{|c|c|c|c|}
\hline Patients Characteristic & PDS (108 patients) & $\begin{array}{l}\text { NACT + IDS (49 } \\
\text { patients) }\end{array}$ & $\begin{array}{c}p \\
\text { value }\end{array}$ \\
\hline Age (years) & $59,3(28-85)$ & $61,2(34-80)$ & 0,197 \\
\hline BMI & $23,8(18,6-34,6)$ & $23,8(17-42,9)$ & 0,424 \\
\hline Parity (number) & $2(0-8)$ & $2(0-5)$ & 0,125 \\
\hline $\begin{array}{l}\text { Age at last period } \\
\text { (years) }\end{array}$ & $50(40-60)$ & $50(42-58)$ & 0,210 \\
\hline preop CA125 (units/mL) & $435,0(14,0-21156,0)$ & $770,0(68,0-36130,0)$ & 0,059 \\
\hline \multicolumn{4}{|l|}{ Menopausal status } \\
\hline $\begin{array}{l}\text { Yes } \\
\text { No }\end{array}$ & $\begin{array}{l}74,1(80) \\
25,9(28) \\
\end{array}$ & $\begin{array}{c}89,8(44) \\
10,2(5)\end{array}$ & 0,033 \\
\hline \multicolumn{4}{|l|}{ ASA } \\
\hline $\begin{array}{l}1 \\
2 \\
3 \\
4 \\
\end{array}$ & $\begin{array}{c}16,7(18) \\
53,7(58) \\
28,7(31) \\
0,9(1) \\
\end{array}$ & $\begin{array}{c}10,2(5) \\
61,2(30) \\
26,5(13) \\
2,0(1) \\
\end{array}$ & 0,559 \\
\hline \multicolumn{4}{|l|}{ Histology } \\
\hline $\begin{array}{l}\text { serous } \\
\text { endometriod } \\
\text { mucinous } \\
\text { clear cells }\end{array}$ & $\begin{array}{c}66,7(72) \\
25,0(27) \\
4,6(5) \\
3,7(4) \\
\end{array}$ & $\begin{array}{c}85,7(42) \\
12,2(6) \\
0(0) \\
2,0(1)\end{array}$ & 0,071 \\
\hline \multicolumn{4}{|l|}{ FIGO STAGE } \\
\hline $\begin{array}{l}\text { III } \\
\text { Illa } \\
\text { Illb } \\
\text { Illc } \\
\text { IV }\end{array}$ & $\begin{array}{c}95.4(103) \\
(8) \\
(25) \\
(70) \\
4.6(5)\end{array}$ & $\begin{array}{c}87.7(43) \\
(1) \\
(0) \\
(42) \\
12.3(6) \\
\end{array}$ & 0,08 \\
\hline \multicolumn{4}{|l|}{ Residual disease } \\
\hline $\begin{array}{l}0 \mathrm{~mm}(\mathrm{RD}=0) \\
1-10 \mathrm{~mm}(\mathrm{RD}=1) \\
>10 \mathrm{~mm}(\mathrm{RD}=2)\end{array}$ & $\begin{array}{l}53,7(58) \\
17,6(19) \\
28,7(31) \\
\end{array}$ & $\begin{array}{c}77,6(38) \\
8,2(4) \\
14,3(7) \\
\end{array}$ & 0,020 \\
\hline \multicolumn{4}{|l|}{ Recurrence } \\
\hline $\begin{array}{l}\text { Yes } \\
\text { No }\end{array}$ & $\begin{array}{l}79,6(86) \\
20,4(22) \\
\end{array}$ & $\begin{array}{c}87,8(43) \\
12,2(6) \\
\end{array}$ & 0,265 \\
\hline \multicolumn{4}{|l|}{ Vital Status } \\
\hline $\begin{array}{l}\text { Alive } \\
\text { Death }\end{array}$ & $\begin{array}{l}35,2(38) \\
64,8(70)\end{array}$ & $\begin{array}{l}22,4(11) \\
77,6(38)\end{array}$ & 0,575 \\
\hline $\begin{array}{l}\text { Length of follow-up } \\
\text { (months) }\end{array}$ & $41,7(1,4-100,0)$ & $34,5(7,6-91,0)$ & 0,21 \\
\hline
\end{tabular}

Continuous variables are expressed as median (range); categorical variables are expressed as percentage (absolute number). IDS = interval-debulking surgery; $\mathrm{NACT}=$ neoadjuvant chemotherapy; PDS = primary debulking surgery

1 versus residual disease $=2$ ) ASA stage (I-II versus III-IV) and number of adjuvant chemotherapy (ACHT) cycles.

\section{Statistical analysis}

Statistical analysis was performed by SPSS software (Chicago, IL, US) for Windows version 19, applying parametric and non-parametric tests when appropriate. We test the approximately normal distribution of sample through the Shapiro-Wilk's test and the visual inspection of the histograms. Due to the non-normal distribution of continuous variables, we analyzed them by $U$ test of MannWhitney; we expressed them as absolute numbers and median (range). Categorical variables have been expressed as percentages and analyzed through the $\chi 2$ test or the Fisher's exact test, when appropriate. Statistically significant differences between treatment arms were defined as $p$ value less than 0.05 .

PFS was defined as the time interval from the date of diagnosis (surgery date for PDS and laparoscopy date for NACT) to the date of the documented first recurrence or progression of disease. If there was no documented recurrence, PFS was calculated from the date of surgery to the date of last follow-up or death, which ever occurred first. Date of progression was determined by serum CA-125 levels and/or computed tomography (CT) scan. OS was defined from the diagnosis date to the death date or last follow-up date.

The Kaplan-Meier method was used to estimate survival curves; for each analysis, the significance of the difference in the unadjusted survival curves was assessed using the log-rank test.

We calculated hazard ratios (HRs) for survival over the entire follow-up period using a Cox proportional hazards model and $95 \%$ confidence intervals (CIs). We entered the following prognostic factors in the multivariable model: type of treatment (PDS versus NACT + IDS), patient age at diagnosis ( $<60$ versus $\geq 60$ years), residual disease after surgical debulking (residual disease $=0$ versus residual disease $=1$ versus residual disease $=2$ ), ASA stage (I-II versus III-IV) and number of ACHT cycles.

\section{Results}

\section{Patients' characteristics}

Over the study period we collected data about 173 patients affected by EOC stage III and IV. We excluded from the analysis a total of 16 patients that after NACT (range 1-8 cycles) did not underwent IDS due to low performance status and progression of the disease. Of the remaining 157 patients, a total of 108 women were included in the PDS group while 49 patients were included in NACT + IDS group. Median age of PDS group was 59.3 years (28-85) versus 61.2 years (34-80) for NACT + IDS group ( $\mathrm{p}=\mathrm{ns})$; median BMI was $23.8 \mathrm{~kg} / \mathrm{m}^{2}(18.6-$ 34.6) for PDS and $23.8 \mathrm{~kg} / \mathrm{m}^{2}$ (17-42.9) for NACT + IDS ( $\mathrm{p}=\mathrm{ns})$; median preoperative CA-125 was 435 $\mathrm{IU} / \mathrm{mL}$ in the PDS group compared with $770 \mathrm{IU} /$ $\mathrm{mL}$ in the NACT group ( $\mathrm{p}=\mathrm{ns})$. Parity, age at last period and ASA classification did not differ signifi- 
cantly between treatment arms ( $\mathrm{p}=\mathrm{ns})$. The $95.4 \%$ (103 women) of patients included in PDS group presented with FIGO stage III and the remaining $4.6 \%$ (5 women) had FIGO stage IV. Similarly, in NACT group $87.7 \%$ of the sample (43 women) was diagnosed as FIGO stage III and only $12.3 \%$ (6 women) had FIGO stage IV. Histologic subtypes differed between groups; endometriod and mucinous were more represented in PDS group; in particular $25.0 \%$ and $4.6 \%$ of patients in PDS versus 12.6 and $0 \%$ in NACT group. Serous subtype was diagnosed in $66.7 \%$ of PDS group and $85.7 \%$ of NACT; clear cell carcinoma was diagnosed in $3.7 \%$ of PDS group versus $2.0 \%$ of NACT group. Patient characteristics grouped by treatment arms are listed in Table 1.

\section{Intraoperative and post-operative data}

In the PDS group a total of 51 (47.2\%) patients underwent diagnostic laparoscopy before debulking surgery. On the contrary, all 49 patients of NACT + IDS group underwent diagnostic laparoscopy before starting NACT. Blood loss, need for transfusion and total number of EC units were significantly higher for PDS group compared to IDS. Median blood loss during surgery was $500 \mathrm{ml}(100-5000)$ for PDS group versus $400 \mathrm{ml}(50-2000)$ in NACT + IDS patients $(p=0.0001)$. As consequence the $40.7 \%$ of patients in PDS arm received blood transfusion compared to $24.5 \%$ of NACT arm ( $p=0.035)$. Also hospitalization length after PDS was significantly higher, with a median of 15 (7-62) days, compared to NACT + IDS, median of $12(5-38)$ days $(\mathrm{p}=0.003)$.

On the contrary, the number of post-operative complications, according to Clavien-Dindo classification system resulted comparable between groups $(p=0.174)$. However, the rate of grade II, III and IV complications was higher in PDS group than in NACT + IDS group.

Finally, the re-operation rate for cancer recurrence did not differ significantly between arms; $24.2 \%$ for PDS group versus $23.8 \%$ for NACT + IDS group $(p=0.174)$. Intraoperative and post-operative data by treatment arm are listed in Table 2 .

\section{Neo-adjuvant and adjuvant chemotherapy data}

Considering NACT + IDS group, the median interval time between diagnostic laparoscopy and the start of NACT was 3 weeks (2-5) and the median interval time between the end of NACT and the IDS was 3 weeks (2-8).
TABLE 2. Patient intra-operative and post-operative data by treatment arm: primary debulking surgery (PDS) $(N=108)$ versus neoadjuvant chemotherapy $(\mathrm{NACT})(\mathrm{N}=$ 49)

\begin{tabular}{|c|c|c|c|}
\hline Variables & $\begin{array}{c}\text { PDS } \\
\text { (108 patients) }\end{array}$ & $\begin{array}{l}\text { NACT + IDS } \\
\text { (49 patients) }\end{array}$ & $p$ value \\
\hline LPS explorative & $47,2(51)$ & $100(49)$ & \\
\hline Blood Loss (ml) & $500,0(100-5000)$ & $400,0(50-2000)$ & 0,0001 \\
\hline EC units & $2(1-23) 44$ patients & 2 (2-3) 12 patients & 0,019 \\
\hline \multicolumn{4}{|l|}{ Transfusion } \\
\hline $\begin{array}{l}\text { Yes } \\
\text { No }\end{array}$ & $\begin{array}{l}40,7(44) \\
59,3(64)\end{array}$ & $\begin{array}{l}24,5(12) \\
75,5(37)\end{array}$ & 0,035 \\
\hline Hospitalization Lenght & $15(7-62)$ & $12(5-38)$ & 0,003 \\
\hline \multicolumn{4}{|l|}{ Post-op complications } \\
\hline $\begin{array}{l}\text { No/Grade I-II } \\
\text { Grade III-IV }\end{array}$ & $\begin{array}{l}77,8(84) \\
22,2(24) \\
\end{array}$ & $\begin{aligned} & 93,9(46) \\
& 6,1(3) \\
&\end{aligned}$ & 0,009 \\
\hline \multicolumn{4}{|c|}{ Reoperation for recurrence } \\
\hline $\begin{array}{l}\text { Yes } \\
\text { No }\end{array}$ & $\begin{array}{l}18,6(16) \\
81,4(70)\end{array}$ & $\begin{array}{l}25,6(11) \\
74,4(32)\end{array}$ & 0,174 \\
\hline
\end{tabular}

Continuous variables are expressed as median (range); categorical variables are expressed as percentage (absolute number). IDS = interval-debulking surgery; NACT = neoadjuvant as percentage (absolute number). IDS = intervo
chemotherapy; PDS = primary debulking surgery

The interval time between surgery and ACHT starting did not differ significantly between treatment arms: median of 4 (3-10) weeks for PDS versus 4 $(2-7)$ weeks for NACT, $(p=n s)$; in the same way also the need for a second line ACHT treatment did not differ between groups $(\mathrm{p}=\mathrm{ns})$.

Concerning the type of ACHT administered the great majority of patients received a combination of carboplatin and paclitaxel (84.8\% in PDS group, $81.3 \%$ in NACT + IDS) followed by carboplatin alone $(8.7 \%$ in PDS group, $8.3 \%$ in NACT + IDS), carboplatin plus doxorubicin (5.4\% in PDS group, $8.3 \%$ in NACT + IDS) and finally carboplatin plus gemcitabine $(1.1 \%$ in PDS group, $2.1 \%$ in NACT + IDS). All data about neo-adjuvant and adjuvant chemotherapy are listed in Table 3.

\section{Residual disease by treatment group}

Patients who received NACT were more likely to have no residual disease compared to patients who underwent PDS $(p=0.02)$. In particular, in PDS group, the $53.7 \%$ of patients resulted completely debulked; the $17.6 \%$ were optimally debulked (for a total of $71.3 \%$ of patients with residual disease $=$ 0 and residual disease $=1$ after PDS) and the 28.7 resulted suboptimally debulked. On the contrary in NACT + IDS group the $77.6 \%$ of the sample resulted completely debulked; the $8.2 \%$ were optimally debulked (for a total of $85.8 \%$ of patients with residual disease $=0$ and residual disease $=1$ 
after IDS) and only $14.3 \%$ resulted suboptimally debulked. All data about residual disease by treatment arm are listed in Table 1.

TABLE 3. Patient data about type of chemotherapy (neoadjuvant chemotherapy [NACT] and adjuvant chemotherapy [ACHT]) and interval time from surgery: primary debulking surgery (PDS) $(N=108)$ versus NACT $(N=49)$

\begin{tabular}{|c|c|c|c|}
\hline Cycles of NACT & \multicolumn{3}{|c|}{$5(3-6)$} \\
\hline \multicolumn{4}{|l|}{ Type of NACT } \\
\hline $\begin{array}{l}\text { Carboplatin } \\
\text { Carboplatin and Paclitaxel } \\
\text { Carboplatin and Doxorubicin }\end{array}$ & \multicolumn{3}{|c|}{$\begin{array}{c}8,2(4) \\
87,8(43) \\
4,1(2) \\
\end{array}$} \\
\hline \multicolumn{4}{|l|}{ Response to NACT } \\
\hline $\begin{array}{l}\text { Complete } \\
\text { Partial }\end{array}$ & \multicolumn{3}{|c|}{$\begin{array}{l}20,4(10) \\
79,6(39)\end{array}$} \\
\hline Interval LPS to NACT (weeks) & \multicolumn{3}{|c|}{$3(2-5)$} \\
\hline $\begin{array}{l}\text { Interval NACT to IDS } \\
\text { (weeks) }\end{array}$ & \multicolumn{3}{|c|}{$3(2-8)$} \\
\hline All Groups & PDS (108 patients) & $\begin{array}{l}\text { NACT + } \\
\text { IDS ( } 49 \\
\text { patients) }\end{array}$ & $\begin{array}{c}p \\
\text { value }\end{array}$ \\
\hline Cycles of ACHT & $6(2-9)$ & $3(2-9)$ & 0,000 \\
\hline \multicolumn{4}{|l|}{ Type of ACHT } \\
\hline $\begin{array}{l}\text { Carboplatin } \\
\text { Carboplatin and Paclitaxel } \\
\text { Carboplatin and Doxorubicin }\end{array}$ & $\begin{array}{c}7,4(8) \\
88,0(95) \\
4,6(5)\end{array}$ & $\begin{array}{c}8,2(4) \\
87,8(43) \\
4,1(2)\end{array}$ & 1,000 \\
\hline $\begin{array}{l}\text { Interval PDS to ACTH and IDS } \\
\text { to ACTH }\end{array}$ & $4(3-10)$ & $4(2-7)$ & 0,147 \\
\hline \multicolumn{4}{|l|}{ Second line ACHT } \\
\hline $\begin{array}{l}\text { Yes } \\
\text { No }\end{array}$ & $\begin{array}{l}78,9(56) \\
21,1(15)\end{array}$ & $\begin{array}{c}79,1(34) \\
20,9(9)\end{array}$ & 0,588 \\
\hline
\end{tabular}

Continuous variables are expressed as median (range); categorical variables are expressed as percentage (absolute number). ACHT = adjuvant chemotherapy; IDS = interval-debulking surgery; $\mathrm{NACT}$ = neoadjuvant chemotherapy; PDS = primary debulking surgery

TABLE 4. Overall survival and progression free survival in patients who underwent primary debulking surgery (PDS) $(N=108)$ and neoadjuvant chemotherapy (NACT) + interval debulking surgery (IDS) ( $N=49$ ); NACT stratified by residual disease (all sample)

\begin{tabular}{lcccc}
\hline & \multicolumn{2}{c}{ PFS } & \multicolumn{2}{c}{ OS } \\
\hline & $\begin{array}{c}\text { Median } \\
\text { (months) }\end{array}$ & $\begin{array}{c}\text { 95\% Cl } \\
\text { (months) }\end{array}$ & $\begin{array}{c}\text { Median } \\
\text { (months) }\end{array}$ & $\begin{array}{c}95 \% \mathrm{Cl} \\
\text { (months) }\end{array}$ \\
\hline PDS & & & & \\
\hline O mm (RD = 0) & 20,7 & $13,2-28,3$ & 54,7 & $40,6-68,7$ \\
1-9 mm (RD = 1) & 11,2 & $10,2-12,2$ & 34,7 & $0,00-71,1$ \\
$>10$ mm (RD = 2) & 13,3 & $10,0-16,5$ & 31,3 & $15,6-47,0$ \\
General & 17,3 & $15,0-19,5$ & 41,3 & $31,2-51,3$ \\
\hline NACT + IDS & & & & \\
\hline O mm (RD = 0) & 19,9 & $16,1-23,7$ & 36,3 & $27,7-44,8$ \\
1-9 mm (RD = 1) & 14,5 & $2,7-26,3$ & 25,6 & $3,9-47,2$ \\
$>10$ mm (RD = 2) & 8,0 & $6,0-9,9$ & 16,1 & $8,1-24,0$ \\
General & 18,3 & $14,9-21,8$ & 34,5 & $26,6-42,4$ \\
\hline
\end{tabular}

Continuous variables are expressed as median (range); categorical variables are expressed as percentage (absolute number). IDS = interval-debulking surgery; $\mathrm{NACT}=$ neoadjuvant chemotherapy; OS = overall survival; PDS = primary debulking surgery; PFS = progression free survival
It is important to underline the fundamental role of explorative laparoscopy (LPS) in predicting the resectability of the tumor. Considering the whole sample (both PDS and NACT + IDS), the 71\% (71 women) of patients who underwent explorative LPS resulted completely debulked (residual disease $=0$ ) compared to the $43.9 \%$ ( 25 women) of patients who did not perform LPS. On the contrary only 13\% (13 women) and 16\% (16 women) of patients who underwent LPS before surgery resulted optimally (residual disease $=1$ ) and sub-optimally debulked (residual disease $=2$ ) respectively, compared to the $17.5 \%$ (10 women) and $38.6 \%$ (22 women) of patients that did not underwent LPS ( $p$ $=0,002$ ). Also considering only PDS patients diagnostic laparoscopy was able to predict significantly the resectability of the tumor. The $64.7 \%$ of patients who underwent diagnostic laparoscopy (33 women) in PDS group resulted completely debulked (residual disease $=0$ ) versus the $43.9 \%$ ( 25 women) who did not receive LPS. Moreover only $17.6 \%$ (9 women) and $17.6 \%$ (9 women) of patients who underwent LPS before surgery resulted optimally (residual disease $=1$ ) and sub-optimally debulked (residual disease $=2$ ) respectively, compared to the $17.5 \%$ (10 women) and $38.6 \%$ (22 women) of patients that did not underwent LPS $(p=0.046)$.

\section{Overall survival and progression free survival}

Considering both treatment arms the median PFS was $17.7(16.0-19.4 ; 95 \% \mathrm{CI})$ months for all patients and the median OS was 37.9 (32.6-43.2; 95\% CI) months for all patients.

The median PFS for patients who underwent PDS was $17.3(15.0-19.5$; 95\% CI) months, the median OS was 41.3 (31.2-51.3 95\% CI) months. The median PFS and OS for patients selected for NACT was $18.3(14.9-21.8 ; 95 \% \mathrm{CI})$ and $34.5(26.6-42.4$; 95\% CI) months, respectively.

Differences in PFS and OS between groups were not statistically significant $(p=0.737$ and $p=0.184$ respectively). The 5-years OS resulted $36.1 \%$ in PDS group versus $26.5 \%$ in NACT + IDS group. All data are presented in Table 4 and Kaplan-Meier curves in Figures 1A and 1B.

Stratifying our arms according to residual disease, we found that patients who underwent NACT + IDS completely debulked (residual disease $=0$ ) experienced a worse median OS (36.3 months; $27.7-44.8 ; 95 \% \mathrm{CI}$ ), even if not statistically significant, when compared to PDS patients completely debulked (residual disease $=0)(\mathrm{OS} 54.7$ 

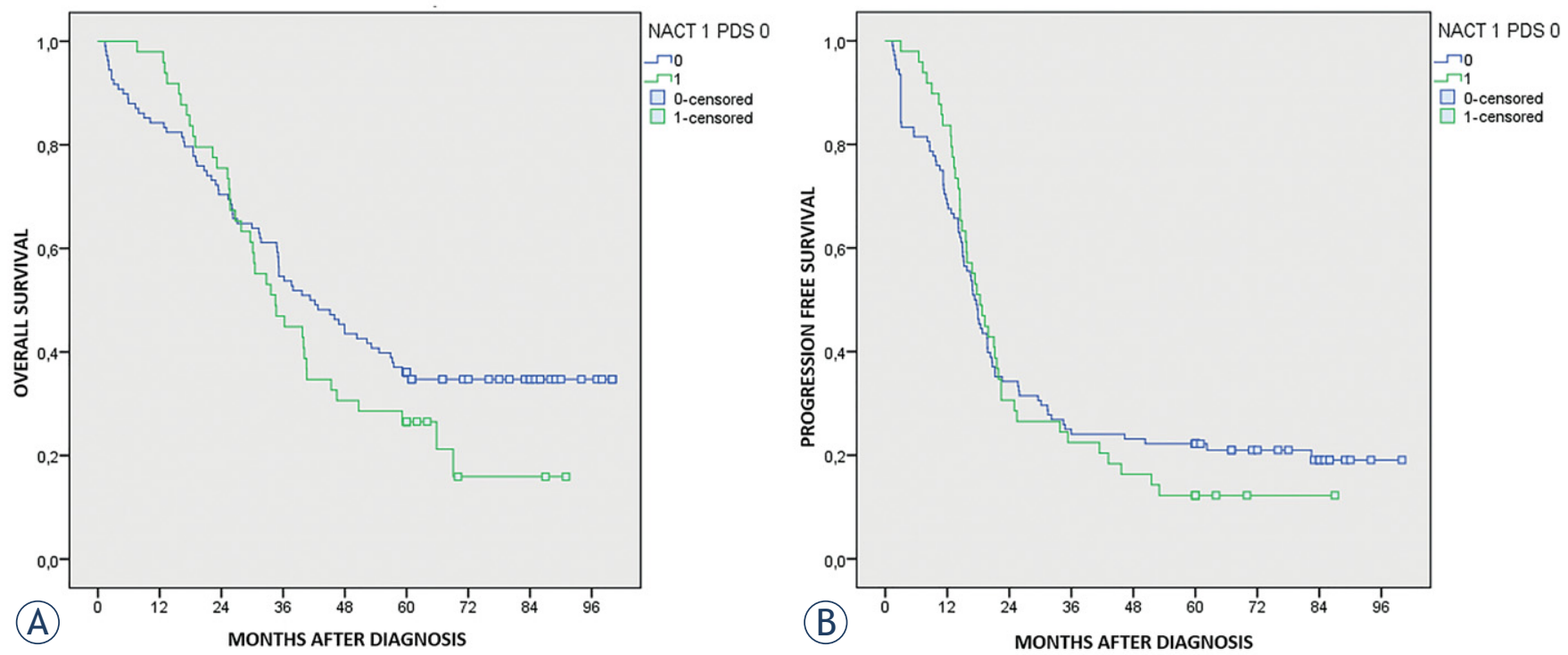

FIGURE 1. (A) Overall survival by treatment arm: primary debulking surgery (PDS) ( $N=108$ ) versus neoadjuvant chemotherapy (NACT) ( $N=49)$. (B) Progression free survival by treatment arm: primary debulking surgery (PDS) ( $N=108$ ) versus neoadjuvant chemotherapy (NACT) ( $N=49$ ).
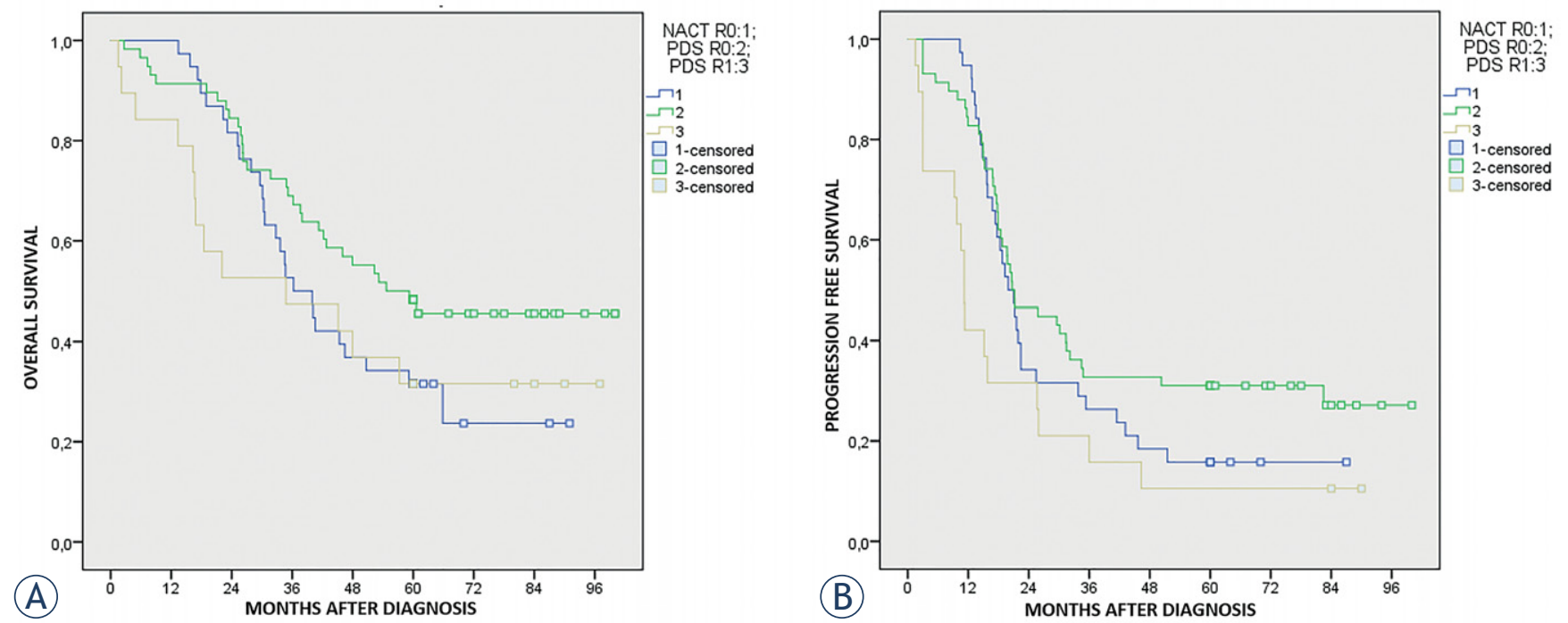

FIGURE 2. (A) Overall survival according the neoadjuvant chemotherapy (NACT) + interval debulking surgery (IDS) with residual disease $=0$ versus primary debulking surgery (PDS) with residual disease $=0$ and PDS with residual disease $=1$. (B) Progression free survival according the neoadjuvant chemotherapy (NACT) + interval debulking surgery (IDS) with residual disease $=0$ versus primary debulking surgery (PDS) with residual disease $=0$ and primary debulking surgery PDS with residual disease $=1$.

months; 40.6-68.7; 95\% CI) ( $\mathrm{p}=0.12)$. The OS between NACT + IDS patients with residual disease $=0$ and PDS patients optimally debulked (residual disease $=1$ ) (OS 34.7 months; 0.00-71.1; 95\% CI) were comparable $(p=0.73)$.

Considering PFS we found that patients who underwent NACT + IDS completely debulked (residual disease $=0$ ) experienced comparable median PFS (19.9 months; 16.1-23.7; 95\% CI) when com- pared to PDS patients completely debulked (residual disease $=0)(20.7$ months; $13.2-28.3 ; 95 \%$ CI $)(p$ $=0.251$ ). The median PFS of PDS patients optimally debulked (residual disease = 1) (11.2 months; 10.2$12.2 ; 95 \% \mathrm{CI}$ ) resulted significantly worse than PFS of NACT + IDS patients completely debulked (residual disease $=0)(p=0.005)$.

The 5 years OS for NACT + IDS patients residual disease $=0$ was $32.6 \%$, for PDS patients residual 
TABLE 5. Univariate and multivariate hazard ratios (HRs) and $95 \%$ confidence intervals (Cls) associated with selected variables, all sample and stratified by treatment group

\begin{tabular}{|c|c|c|c|c|}
\hline Variables & $\begin{array}{c}\text { Univariate HR } \\
(95 \% \mathrm{Cl})\end{array}$ & $p$ & $\begin{array}{c}\text { Multivariate HR } \\
(95 \% \mathrm{Cl})\end{array}$ & p \\
\hline \multicolumn{5}{|l|}{ All sample (157) } \\
\hline \multicolumn{5}{|l|}{ Treatment group } \\
\hline PDS & 1.00 (reference) & & & \\
\hline $\mathrm{NACT}+\mathrm{IDS}$ & $1,34(0,90-1,99)$ & 0,14 & $1,45(0,87-2,42)$ & 0,14 \\
\hline \multicolumn{5}{|l|}{ Residual Disease } \\
\hline $0 \mathrm{~mm}(\mathrm{RD}=0)$ & 1.00 (reference) & & & \\
\hline $1-9 \mathrm{~mm}(\mathrm{RD}=1)$ & $1,51(0,88-2,60)$ & 0,13 & $1,66(0,96-2,82)$ & 0,06 \\
\hline$>10 \mathrm{~mm}(\mathrm{RD}=2)$ & $2,29(1,49-3,53)$ & 0,0001 & $2,82(1,79-4,46)$ & 0,0001 \\
\hline \multicolumn{5}{|l|}{ Age } \\
\hline$<60$ & 1.00 (reference) & & & \\
\hline$\geq 60$ & $1,33(0,91-1,95)$ & 0,13 & $0,91(0,61-1,38)$ & 0,68 \\
\hline \multicolumn{5}{|l|}{ ASA } \\
\hline I- II & 1.00 (reference) & & & \\
\hline III - IV & $2,29(1,53-3,41)$ & 0,0001 & $2,21(1,44-3,40)$ & 0,0001 \\
\hline ACHT cycles & $0,94(0,82-1,08)$ & 0,42 & $0,99(0,85-1,16)$ & 0,96 \\
\hline \multicolumn{5}{|l|}{ PDS group } \\
\hline \multicolumn{5}{|l|}{ Residual Disease } \\
\hline $0 \mathrm{~mm}(\mathrm{RD}=0)$ & 1.00 (reference) & & & \\
\hline $1-9 \mathrm{~mm}(\mathrm{RD}=1)$ & $1,70(0,89-3,26)$ & 0,10 & $2,12(1,08-4,15)$ & 0,028 \\
\hline$>10 \mathrm{~mm}(\mathrm{RD}=2)$ & $2,37(1,40-4,0)$ & 0,001 & $3,12(1,80-5,41)$ & 0,00005 \\
\hline \multicolumn{5}{|l|}{ Age } \\
\hline$<60$ & 1.00 (reference) & & & \\
\hline$\geq 60$ & $1,49(0,93-2,39)$ & 0,09 & $0,81(0,48-1,37)$ & 0,44 \\
\hline \multicolumn{5}{|l|}{ ASA } \\
\hline I- II & 1.00 (reference) & & & \\
\hline III - IV & $3,44(2,08-5,68)$ & 0,001 & $4,40(2,50-7,75)$ & 0,0001 \\
\hline ACHT cycles & $0,77(0,51-1,16)$ & 0,22 & $0,70(0,53-0,93)$ & 0,015 \\
\hline \multicolumn{5}{|l|}{ NACT + IDS group } \\
\hline \multicolumn{5}{|l|}{ Residual Disease } \\
\hline $0 \mathrm{~mm}(\mathrm{RD}=0)$ & 1.00 (reference) & & & \\
\hline $1-9 \mathrm{~mm}(\mathrm{RD}=1)$ & $1,38(0,47-3,98)$ & 0,55 & $1,53(0,52-4,52)$ & 0,43 \\
\hline$>10 \mathrm{~mm}(\mathrm{RD}=2)$ & $\begin{array}{c}4,92(2,04-11 \\
88)\end{array}$ & 0,0001 & $6,67(2,43-18,33)$ & 0,0001 \\
\hline \multicolumn{5}{|l|}{ Age } \\
\hline \multicolumn{5}{|l|}{$<60$} \\
\hline$\geq 60$ & $0,95(0,50-1,81)$ & 0,88 & $0,71(0,35-1,47)$ & 0,36 \\
\hline \multicolumn{5}{|l|}{ ASA } \\
\hline \multicolumn{5}{|l|}{$\mid-\|$} \\
\hline III - IV & $0,99(0,48-2,01)$ & 0,97 & $0,77(0,35-1,69)$ & 0,52 \\
\hline ACHT cycles & $1,01(0,85-1,19)$ & 0,89 & $1,03(0,86-1,23)$ & 0,70 \\
\hline
\end{tabular}

Residual disease, age class, ASA score were included in the multivariate analysis. ACHT = adjuvant chemotherapy; $\mathrm{ASA}=$ American Society of Anesthesiologists; IDS = interval-debulking surgery; $\mathrm{NACT}=$ neoadjuvant chemotherapy; PDS = primary debulking surgery disease $=0$ was $48.3 \%$ and finally for PDS patients residual disease $=1$ was $31.6 \%$. The 5 years PFS for NACT + IDS patients residual disease $=0$ was $15.8 \%$, for PDS patients residual disease $=0$ was $31.0 \%$ and finally for PDS patients residual disease $=1$ was $10.5 \%$. See Kaplan-Meier curves in Figures 2A and 2B. All data about OS and PFS stratified by residual disease are summarized in Table 4.

In our sample there were differences between groups in the distribution of histological type (the great majority of endometriod and all mucinous were included in PDS arm), even if we considered only high grade tumor, this fact could be a potential source of bias. Due to this fact, we decided to repeat vital analysis excluding endometriod and mucinous subtypes.

As expected, also excluding these subtype, the median PFS and OS resulted comparable between groups ( $p=0.634$ and $p=0.541$ respectively). The median PFS and OS for patients selected for PDS was 16.8 (14.6-19.0; 95\% CI) and 39.6 (28.9-50.3; $95 \% \mathrm{CI}$ ) months respectively. The median PFS and OS for patients selected for NACT + IDS was 19.3 (15.0-23.5; 95\% CI) and 36.3 (27.0-45.5; 95\% CI) months respectively. See Kaplan-Meier curves in Figures $3 \mathrm{~A}$ and $3 \mathrm{C}$.

Also in this case stratifying our arms according to residual disease, we found that NACT + IDS patients completely debulked (residual disease $=$ 0) showed a worse median OS (39.9 months; 31.348.6; 95\% CI), although not significant, compared to PDS patients completely debulked (residual disease $=0)(47.9$ months; $34.5-61.3$; 95\% CI $)(p=$ $0.434)$. On the contrary, median OS of NACT + IDS completely debulked (residual disease $=0$ ) was better than median OS of PDS patients optimally debulked (residual disease $=1$ ) (34.7 months; 0.0076.9; $95 \% \mathrm{CI})$, but still not significant ( $\mathrm{p}=0.656$ ).

In the same way, median PFS of NACT + IDS patients completely debulked (residual disease $=0$ ) resulted comparable (21.0 months, 17.7-24.3; 95\% CI) to PDS patients with no residual disease (residual disease $=0)(19.7$ months, $16.3-23.1 ; 95 \%$ CI $)$ $(p=0.904)$. On the contrary, median PFS in PDS patients optimally debulked (residual disease $=$ 1) (10.6 months; $7.8-13.3$; 95\% CI) resulted significantly worse than NACT + IDS patients completely debulked (residual disease $=0)(p=0.012)$. See Kaplan-Meier curves in Figures 4A and 4B. While the 5 years OS for NACT + IDS patients residual disease $=0$ was $35.3 \%$, for PDS patients residual disease $=0$ was $38.5 \%$ OS.

We conducted a multivariate survival analysis for the whole sample and according to treatment 

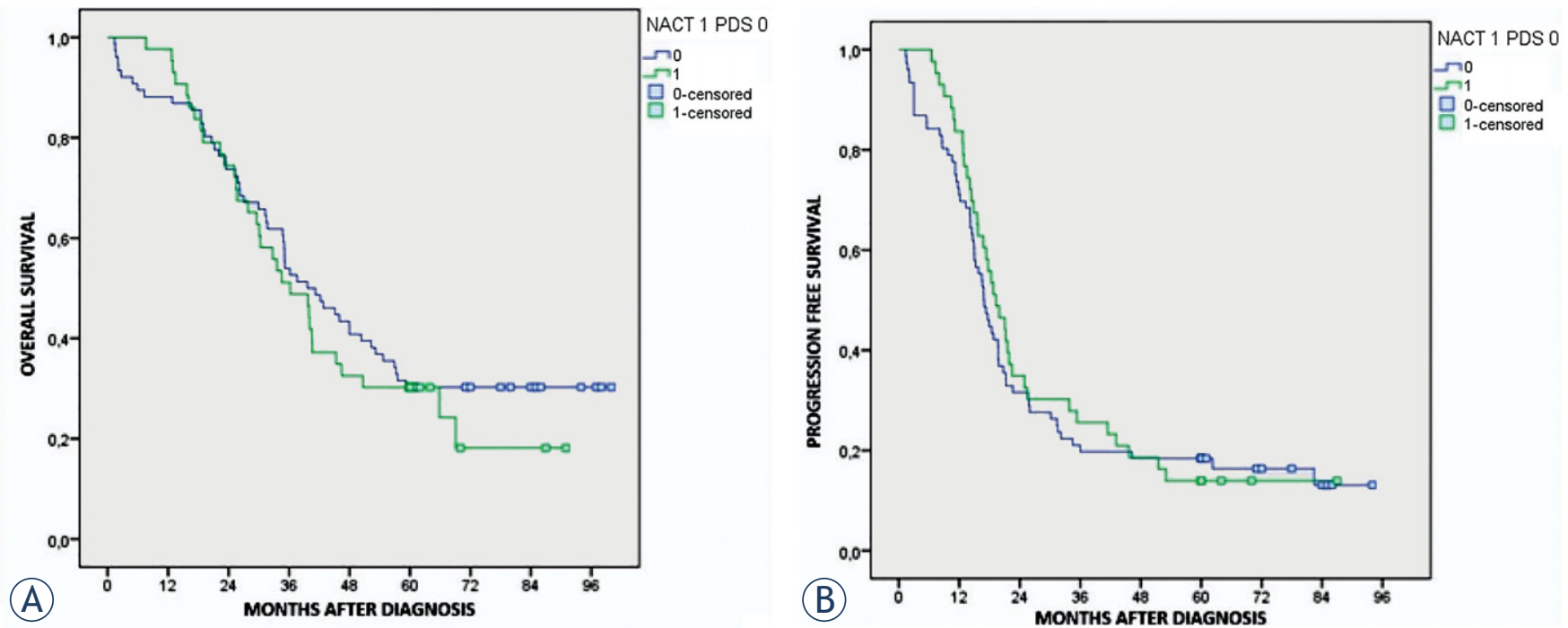

FIGURE 3. (A) Overall survival by treatment excluding endometriod and mucinous histotype. (B) Progression free survival by treatment excluding endometriod and mucinous histotype.
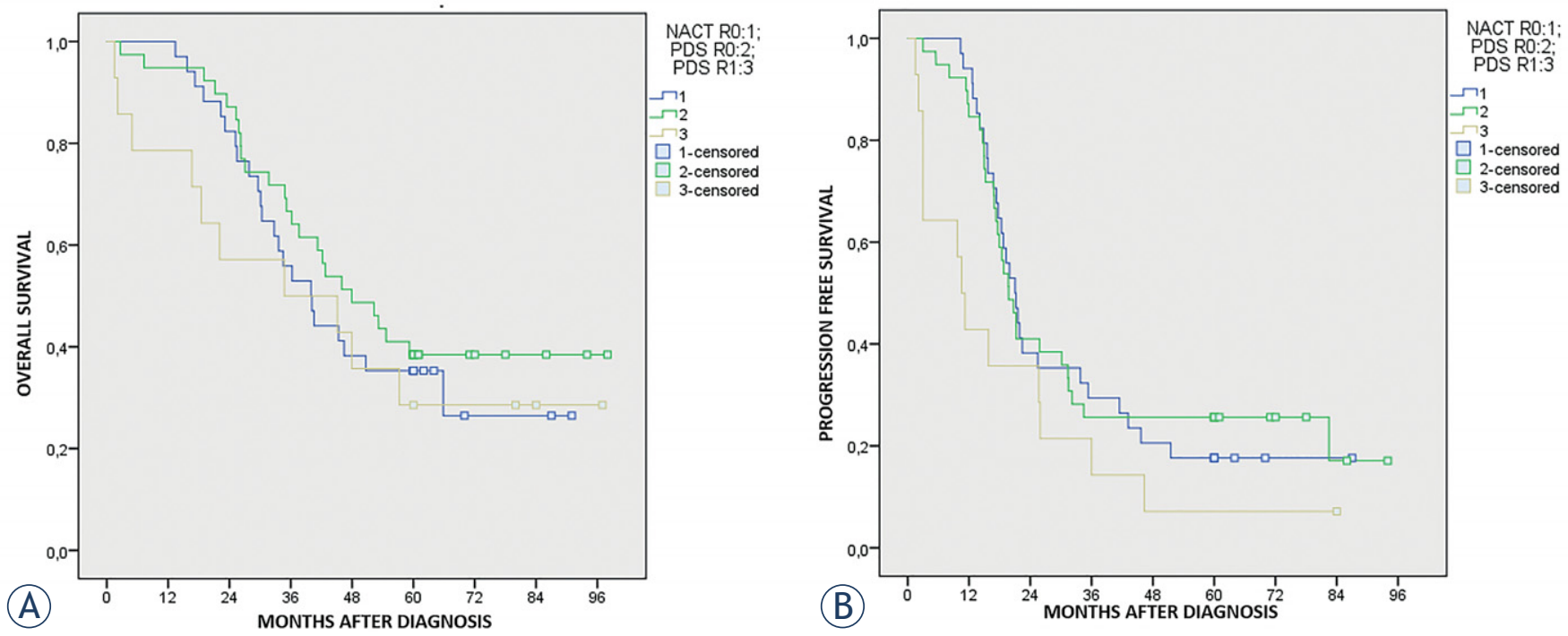

FIGURE 4. (A) Overall survival according the neoadjuvant chemotherapy (NACT) + interval debulking surgery (IDS) with residual disease $=0$ versus primary debulking surgery (PDS) with residual disease $=0$ and PDS with residual disease $=1$ (excluding endometriod and mucinous histotype). (B) Progression free survival according the neoadjuvant chemotherapy (NACT) + interval debulking surgery (IDS) with residual disease $=0$ versus primary debulking surgery (PDS) with residual disease $=0$ and PDS with residual disease $=1$ (excluding endometriod and mucinous histotype).

arms. Considering both the whole sample and treatments arms, the presence of residual disease after debulking surgery and pre-operative ASA score > II were significant predictors of survival. On the contrary, age lower than 60 years did not relate with better OS. The number of cycles of ACHT was significantly associated with survival only for PDS arm in multivariate model. Univariate and multivariate analysis are presented in Table 5 .

\section{Discussion}

\section{General considerations}

It is widely confirmed that the amount of residual disease at the time of surgery is the pivotal determinant of outcome in patients affected by advanced stage EOC. ${ }^{20}$ Du Bois et al., analyzing the results of three randomized trials, demonstrated that patients with no residual disease showed a 
significant better OS and PFS when compared both to optimally and sub-optimally debulked patients. ${ }^{4}$ Therefore, it is now accepted that the goal of cytoreductive surgery changed from a residual disease of 1-2 cm to the complete resection of macroscopically visible tumor. ${ }^{20,21}$

However, in advanced stage EOC the rate of complete debulking is generally estimated lower than $50 \% .^{9-11}$ The reasons for suboptimal debulking may be related to large intra-abdominal extension of the tumor, localization in critical anatomical site, medical comorbidities, advanced age and poor oncological experience of surgeons. ${ }^{4,22,23}$ All such variables are of crucial importance to understand the reasons that led the paradigm shift, in selected cases, from standard PDS approach to alternative therapeutic options like NACT + IDS treatment. ${ }^{10,15,16}$

The rationale of administering NACT before dubulking surgery is to reduce disease spread in abdominal pelvic cavity, in order to increase the probability to perform a subsequent complete debulking with less aggressive surgery and lower post-operative complications. ${ }^{20}$

In our series we found that the $77.6 \%$ of patients in NACT + IDS arm showed no macroscopic residual disease after surgery versus the $53.7 \%$ of PDS arm. These data certainly confirmed the principle that after NACT administration the rate of complete or optimal debulking is strongly higher. Recent series are in line with our results; in the recent paper by Mueller et al. a $47 \%$ of PDS and $55 \%$ of NACT patients underwent complete gross resection. ${ }^{10}$ Also May et al. described a residual disease $=0$ in $35.2 \%$ of PDS patients versus $42.4 \%$ of NACT group. ${ }^{9,10}$ In addition, also the CHORUS randomized trial described a better rate of residual disease $=0$ after IDS than after PDS, even if the 17\% of complete debulking after PDS and of 39\% after IDS is drastically lower if compared to our results but also to other series. ${ }^{9,10,11}$

Concerning the post-operative complications rate, we confirmed data by previous trials; we found a significant higher rate of grade 3 and 4 complications in PDS group compared to NACT. Both two published randomized trials reported higher rate of severe post-operative complications and mortality in primary surgery group when compared to NACT + IDS arm. ${ }^{12,13}$

However, it is interesting to highlight that higher rate of complete debulking $(R=0)$, as well as the lower rate of surgery-related complications in NACT + IDS group, did not result in increased OS in large majority of studies; differently, OS was equally comparable or even inferior to PDS arm. In the first published randomized trial Vergote $e t$ al. analyzing 632 patients treated by PDS or NACT + IDS found no differences in OS (29 months in PDS and 30 months in NACT) between the treatments groups. ${ }^{13}$ Similarly, in experience by Kehoe et al, the comparison between 276 patients assigned to primary surgery versus 274 assigned to primary chemotherapy showed no differences in term of OS (22.6 months in PDS and 24.1 months in NACT) and PFS (10.7 months in PDS and 12.0 months in NACT $).{ }^{12}$

Stating these data, both Authors concluded that giving primary chemotherapy before surgery is an acceptable standard of care for women with advanced stage EOC. However, different concerns have been raised regarding the reproducibility of these results. ${ }^{14}$ Drawbacks include the above mentioned poor rate of surgical radicality compared to other studies; the low-median operating time (120-165 minutes) and finally, most importantly, the very low OS and PFS reported. ${ }^{9,10}$ Starting from these limitations of evidence quality from RCTs, the debate on the ideal management of advanced EOC is still open and a comprehensive evaluation of literature should take in account also data from lowly powered studies; at this regard, different authors published some retrospective analysis that reached opposite results in comparison to RCTs. In a recent paper May et al. described a better five-years OS in primary surgery group $(39 \%)$ compared to NACT + IDS arm (27\%). ${ }^{9}$ Also Mulller et al. in their analysis of a cohort of 586 patients demonstrated that PDS patients had significantly higher median OS and PFS (71.7 and 21.7 months respectively) in comparison to NACT patients (42.9 and 13.9 months respectively). ${ }^{10}$ From the data analysis of American National Cancer Database, including women with primary EOC with less than 70 years and without comorbidities, Rauh-Hain et al. described a median OS of 37.3 months in the PDS group and 32.1 months in the NACT group $(\mathrm{p}<0.001) .{ }^{24}$ Finally, also Rosen et al. and Kessous et al. reached similar results. ${ }^{11,12}$ These different findings in comparison to RCTs may be related to several factors, such as the retrospective design of the studies and the selection bias related to patients included in NACT group, who may be affected by greater spread of disease and lower performance status at the time of study inclusion.

Moreover, another important factor to be considered is that the poor outcome of NACT patients (also with lower residual disease) could be related to own tumor biology, potentially related with 
lower chemosensitivity in comparison to patients in which a complete primary debulking can be obtained. In particular, different retrospective series suggested a strong association between tumor volume, suboptimal cytoreduction and NACT + IDS with platinum resistance..$^{24-26}$ The most widely accepted hypothesis implicates the outgrowth of resistant clones, which are usually present at low rates before initial treatment and are greater in large tumor volumes treated with platinum compounds. ${ }^{27}$

\section{Main findings}

In our series, considering the whole sample, although the higher rate of no residual disease in NACT + IDS arm and of surgical complications of PDS arm, we found a lower OS, but not statistically significant, in NACT + IDS arm (34.4 months) compared to PDS arm (41.3 months). PFS resulted comparable between groups (17.3 months in PDS versus 18.3 months in NACT arm). Also excluding endometrioid and mucinous subtype, in order to decrease as much as possible the selection bias, the trend resulted comparable (OS of 39.6 months in PDS versus 36.3 in NACT arm; PFS of 16.8 months in PDS versus 19.3 months in NACT arm). Our results are in line with retrospective series by Lim et al. and Bian et al. but also with ERTOC and CHORUS randomized trials. ${ }^{12,13,28,29}$

Considering that the pivotal factor that drives surgical action is the possibility to obtain a complete/optimal cytoreduction, we compared the survival in both arms stratifying data according to residual disease (Table 4). We found better OS, even if not statistically significant, in patients with no residual disease included in PDS arm compared to NACT + IDS arm. Median OS of patients of PDS arm optimally debulked was comparable to NACT + IDS with no residual disease. The PFS of patients with no residual disease was comparable in both groups; however, PDS patients optimally debulked experienced a significant lower PFS of about 9 months if compared to NACT + IDS patients completely debulked.

As in our sample endometrioid and mucinous subtypes were mostly represented in PDS arm, we decided to repeat the analysis excluding these subgroups. We took this decision basing both on statistical reasons, in order to eliminate selection bias between groups, and on biological reasons. Indeed endometrioid cancer is generally associated with better outcome compared to other histology due to its intrinsic biological behavior and to its asso- ciation with lower grading and staging. ${ }^{30}$ Similarly, mucinous subtypes are associated to a good prognosis in case of lower stage, but to worst prognosis (due to its intrinsic biological features of chemo-resistance) if compared to other histological types in case of advanced stage. ${ }^{31}$ Interestingly, also excluding these cases we found no change in trends of OS and PFS previously reported.

Therefore, from our analysis, we confirm a non-superiority of NACT + IDS in term of median OS and PFS when compared to standard PDS approach. However, stratifying data based on residual disease after surgery we found that benefits of PDS approach are maximized when residual disease $=0$ was attained. The benefit of PDS resulted comparable between optimal debulked PDS patients and complete dubulked NACT patients in term of OS. The median PFS was comparable between treatment arms in case of residual disease $=$ 0 ; on the contrary benefit of a PDS approach with residual disease $=1$ was drastically lower, when compared to NACT + IDS with residual disease $=0$.

This data is of crucial importance for patients because a less aggressive surgery and the interval time without disease could be considered surrogate measurement of patient's quality of life. Certainly, also in case of NACT administration maximum efforts must be made to obtain no residual disease after IDS; in a recent report by Marchetti et al., patients who did not undergo IDS after NACT showed a median OS of 18 months that is similar to median OS of our patients with residual disease $=2$ after IDS. ${ }^{32}$ Therefore, the selection of patients suitable for IDS was a fundamental step to improve survivals and to avoid unnecessary surgery that can only result in a decrease of patient's quality of life.

Different approach has been tested to predict the resectability of tumor like CT, MR and positron emission tomography imaging. However, recent studies point a highly valuable role for laparoscopic Fagotti score to assess the feasibility of complete/optimal cytoreduction. ${ }^{7}$ In our sample, all NACT patients received laparoscopy before starting chemotherapy; the decision was not based on a defined laparoscopic score but on experience of gynecologic oncologist surgeon. In our series we confirmed the value of diagnostic laparoscopy for resectability assessment; patients of PDS arm who underwent laparoscopy before surgery showed a significant higher rate of complete debulking compared to patients who did not undergo laparoscopy. In our opinion, laparoscopic assessment before PDS or IDS should became one of the fundamental diagnostic steps to drive treatment decision. 
According to different papers we found in univariate and multivariate model that both in PDS and NACT + IDS arms the most important predictor of survival was represented by the residual disease after cytoreductive surgery. ${ }^{9,10}$ Moreover, according to Gill et al. experience we found also that an ASA score $>2$ was strongly associated with survival in both univariate and multivariate model. ${ }^{33}$ Concerning adjuvant chemotherapy cycles we found no differences in both univariate and multivariate models in NACT arm, but we found an association with better survival in multivariate analysis in PDS arm.

\section{Limits of the study}

Our study is affected by different limitations; the main potential source of bias is related to patients' selection, as primary treatment choice (between PDS and NACT) was based on the spread of the disease and patients' health status; the retrospective design of the study did not allow overcoming this bias.

Secondly, the pre-operative laparoscopic assessment of abdominal cavity was not based on a pre-defined score but on single operator experience; however, the choice of primary treatment was made by a Multidisciplinary Group (including gynecologic oncologists, medical oncologists, radiologists and pathologists) basing on criteria that are similar to other studies. Finally, we included in the analysis only patients who underwent IDS after chemotherapy, excluding 16 patients that did not undergo surgery due to disease progression or medical conditions, potentially leading to an overstatement of NACT + IDS patient's survivals.

Main points of strength of our study are related to rigorous data collection methodology and strict inclusion criteria; all information were collected from our electronic hospital records, which are compiled by clinicians at each step of patient's treatment, representing certainly a guarantee of the completeness and correctness of the data reported. We included exclusively patients referred from diagnosis to treatment to our oncological institute, excluding any sources of bias related to heterogeneous surgical choices and procedures.

Moreover, we selected only high grade and advanced stage EOC, minimizing the heterogeneity related to variable disease spread and stage-related prognosis; additionally, all patients received adjuvant chemotherapy based on platinum agents. A sensitivity analysis was also performed in order to exclude any sources of selection bias potentially related to different outcomes of specific histological subtypes of ovarian cancer. Finally, both study groups resulted comparable in term of general features and length of follow-up (at least 60 months). Despite the different limitations of our study, all these points of strength seemingly confer adequate accuracy to our survival estimates.

\section{Conclusions}

In conclusion, according to recent literature, we reaffirm the non-superiority of NACT + IDS compared to PDS approach for the treatment of EOC, even if NACT + IDS treatment is associated with significant lower post-operative complications. In order to maximize survival and to ensure a good quality of life, it is mandatory to define the most effective treatment for advanced EOC based on pre-operative conditions, and most importantly according to potential resectability of tumor. Stating that the goal of advanced stage EOC surgery is to reach complete cytoreduction, more efforts are needed to allow an adequate selection of patients that can benefit from PDS or IDS surgery, avoiding unfavorable procedures. In this light, the laparoscopic primary assessment of tumor extension seems to be reliable in estimating tumor resectability and may potentially represent a valuable strategy for the decision making between primary and interval debulking surgery. However, further studies are needed to confirm the rationale of the use of primary diagnostic laparoscopy as the standard of care in all oncological institutions.

We confirmed the value of primary PDS approach for the treatment of high grade, advanced stage EOC, even if OS is maximized only if no residual disease is attained. If the chances to reach a complete debulking at PDS are low, NACT + IDS approach (with the goal of subsequent residual disease $=0$ ) must be considered because even if we did not evidence benefits in term of OS we found benefits in term of PFS (if compared to PDS with residual disease $=1$ ) that could be related to an increase quality of life. However, if the chances to obtain a complete or at least optimal debulking after standard cycles of NACT are still low, probably subsequent IDS will not be useful to increase patient's survival. 


\section{References}

1. Siegel RL, Miller KD, Jemal A. Cancer statistics, 2017. CA Cancer J Clin 2017; 67: 7-30. doi: 10.3322/caac.21387

2. Ataseven B, Chiva LM, Harter P, Gonzalez-Martin A, du Bois A. FIGO stage IV epithelial ovarian, fallopian tube and peritoneal cancer revisited. Gynecol Oncol 2016; 142: 597-607. doi: 10.1016/j.ygyno.2016.06.013

3. Kumar L, Pramanik R, Kumar S, Bhatla N, Malik S. Neoadjuvant chemotherapy in gynaecological cancers - Implications for staging. Best Pract Res Clin Obstet Gynaecol 2015; 29: 790-801. doi: 10.1016/j.bpobgyn.2015.02.008

4. du Bois A, Reuss A, Pujade-Lauraine E, Harter P, Ray-Coquard I, Pfisterer J. Role of surgical outcome as prognostic factor in advanced epithelial ovarian cancer: a combined exploratory analysis of 3 prospectively randomized phase 3 multicenter trials: by the Arbeitsgemeinschaft Gynaekologische Onkologie Studiengruppe Ovarialkarzinom (AGO-OVAR) and the Groupe d'Investigateurs Nationaux Pour les Etudes des Cancers de l'Ovaire (GINECO). Cancer 2009 15; 115: 1234-44. doi: 10.1002/cncr.24149

5. Kessous R, Laskov I, Abitbol J, Bitharas J, Yasmeen A, Salvador S, et al. Clinical outcome of neoadjuvant chemotherapy for advanced ovarian cancer. Gynecol Oncol 2017; 144: 474-9. doi: 10.1016/j.ygyno.2016.12.017

6. Liu Z, Beach JA, Agadjanian H, Jia D, Aspuria PJ, Karlan BY, et al. Suboptimal cytoreduction in ovarian carcinoma is associated with molecular pathways characteristic of increased stromal activation. Gynecol Oncol 2015; 139: 394-400. doi: 10.1016/j.ygyno.2015.08.026

7. Gómez-Hidalgo NR, Martinez-Cannon BA, Nick AM, Lu KH, Sood AK, Coleman RL, et al. Predictors of optimal cytoreduction in patients with newly diagnosed advanced-stage epithelial ovarian cancer: Time to incorporate laparoscopic assessment into the standard of care. Gynecol Oncol 2015; 137: 553-8. doi: 10.1016/j.ygyno.2015.03.049

8. Melamed A, Hinchcliff EM, Clemmer JT, Bregar AJ, Uppal S, Bostock I, et al. Trends in the use of neoadjuvant chemotherapy for advanced ovarian cancer in the United States. Gynecol Oncol 2016; 143: 236-40. doi: 10.1016/j. ygyno.2016.09.002

9. May T, Comeau R, Sun P, Kotsopoulos J, Narod SA, Rosen B, et al. A Comparison of Survival Outcomes in Advanced Serous Ovarian Cancer Patients Treated With Primary Debulking Surgery Versus Neoadjuvant Chemotherapy. Int J Gynecol Cancer 2017; 27: 668-74. doi: 10.1097/ IGC.0000000000000946

10. Mueller JJ, Zhou QC, lasonos A, O'Cearbhaill RE, Alvi FA, El Haraki A, et al. Neoadjuvant chemotherapy and primary debulking surgery utilization for advanced-stage ovarian cancer at a comprehensive cancer center. Gynecol Oncol 2016; 140: 436-42. doi: 10.1016/j.ygyno.2016.01.008

11. Rosen B, Laframboise S, Ferguson S, Dodge J, Bernardini M, Murphy J, et al. The impacts of neoadjuvant chemotherapy and of debulking surgery on survival from advanced ovarian cancer. Gynecol Oncol 2014; 134: 462-7. doi: 10.1016/j.ygyno.2014.07.004

12. Kehoe S, Hook J, Nankivell M, Jayson GC, Kitchener H, Lopes T, et al. Primary chemotherapy versus primary surgery for newly diagnosed advanced ovarian cancer (CHORUS): an open-label, randomised, controlled, non-inferiority trial. Lancet 2015; 386: 249-57. doi: 10.1016/S0140-6736(14)62223-6

13. Vergote I, Tropé CG, Amant F, Kristensen GB, Ehlen T, Johnson N, et al. European Organization for Research and Treatment of CancerGynaecological Cancer Group; NCIC Clinical Trials Group. Neoadjuvant chemotherapy or primary surgery in stage IIIC or IV ovarian cancer. N Engl J Med 2010; 363: 943-53. doi: 10.1056/NEJMoa0908806

14. Mahner S, Trillsch F, Chi D, Harter P, Pfisterer J, Hilpert F, et al. Neoadjuvan chemotherapy in ovarian cancer revisited. Ann Oncol 2016; 27 (Suppl 1): i30-2. doi: 10.1093/annonc/mdw092

15. Cornelis S, Van Calster B, Amant F, Leunen K, van der Zee AG, Vergote I. Role of neoadjuvant chemotherapy in the management of stage IIIC-IV ovarian cancer: survey results from the members of the European Society of Gynecological Oncology. Int J Gynecol Cancer 2012; 22: 407-16. doi: 10.1097/IGC.0b013e31823ea1d8

16. Dewdney SB, Rimel BJ, Reinhart AJ, Kizer NT, Brooks RA, Massad LS, et al. The role of neoadjuvant chemotherapy in the management of patients with advanced stage ovarian cancer: survey results from members of the Society of Gynecologic Oncologists. Gynecol Oncol 2010; 119: 18-21. doi: 10.1016/j. ygyno.2010.06.021
17. Chan YM, Ng TY, Ngan HY, Wong LC. Quality of life in women treated with neoadjuvant chemotherapy for advanced ovarian cancer: a prospective longitudinal study. Gynecol Oncol 2003; 88: 9-16. doi:10.1006/gyno.2002.6849

18. Greimel E, Kristensen GB, van der Burg ME, Coronado P, Rustin G, del Rio AS, et al. European Organization for Research and Treatment of Cancer - Gynaecological Cancer Group and NCIC Clinical Trials Group. Quality of life of advanced ovarian cancer patients in the randomized phase III study comparing primary debulking surgery versus neo-adjuvant chemotherapy. Gynecol Oncol 2013; 131: 437-44. doi: 10.1016/j.ygyno.2013.08.014

19. Clavien PA, Barkun J, de Oliveira ML, Vauthey JN, Dindo D, Schulick RD, et al The Clavien-Dindo classification of surgical complications: five-year experience. Ann Surg 2009; 250: 187-96. doi: 10.1097/SLA.0b013e3181b13ca2

20. Karam A, Ledermann JA, Kim JW, Sehouli J, Lu K, Gourley C, et al. Fifth Ovarian Cancer Consensus Conference of the Gynecologic Cancer InterGroup: first-line interventions. Ann Oncol 2017; 28: 711-7. doi: 10.1093/annonc/mdx011

21. Chang SJ, Hodeib M, Chang J, Bristow RE. Survival impact of complete cytoreduction to no gross residual disease for advanced-stage ovarian cancer: a meta-analysis. Gynecol Oncol 2013; 130: 493-8. doi: 10.1016/j. ygyno.2013.05.040

22. Brand AH. Ovarian cancer debulking surgery: a survey of practice in Australia and New Zealand. Int J Gynecol Cancer 2011; 21: 30-5. doi: 10.1097/IGC.0b013e318205fb4f

23. Dahm-Kähler P, Palmqvist C, Staf C, Holmberg E, Johannesson L. Centralized primary care of advanced ovarian cancer improves complete cytoreduction and survival - A population-based cohort study. Gynecol Oncol 2016; 142: 211-6. doi: 10.1016/j.ygyno.2016.05.025

24. Rauh-Hain JA, Melamed A, Wright A, Gockley A, Clemmer JT, Schorge JO, et al. Overall Survival Following Neoadjuvant Chemotherapy vs Primary Cytoreductive Surgery in Women With Epithelial Ovarian Cancer: Analysis of the National Cancer Database. JAMA Oncol 2017; 3: 76-82. doi: 10.1001/ jamaoncol.2016.4411

25. Petrillo M, Ferrandina G, Fagotti A, Vizzielli G, Margariti PA, Pedone AL, et al. Timing and pattern of recurrence in ovarian cancer patients with high tumor dissemination treated with primary debulking surgery versus neoadjuvant chemotherapy. Ann Surg Oncol 2013; 20: 3955-60. doi: 10.1245/ s10434-013-3091-6

26. da Costa AA, Valadares CV, Baiocchi G, Mantoan H, Saito A, Sanches S, et al. Neoadjuvant Chemotherapy Followed by Interval Debulking Surgery and the Risk of Platinum Resistance in Epithelial Ovarian Cancer. Ann Surg Oncol 2015; 22 (Suppl 3): S971-8. doi: 10.1245/s10434-015-4623-z

27. Cooke SL, Brenton JD. Evolution of platinum resistance in high-grade serous ovarian cancer. Lancet Oncol 2011; 12: 1169-74. doi: 10.1016/S14702045(11)70123-1

28. Bian C, Yao K, Li L, Yi T, Zhao X. Primary debulking surgery vs. neoadjuvant chemotherapy followed by interval debulking surgery for patients with advanced ovarian cancer. Arch Gynecol Obstet 2016; 293: 63-8. doi: 10.1007/ s00404-015-3813-z

29. Lim MC, Yoo HJ, Song YJ, Seo SS, Kang S, Kim SH, et al. Survival outcomes after extensive cytoreductive surgery and selective neoadjuvant chemotherapy according to institutional criteria in bulky stage IIIC and IV epithelial ovarian cancer. J Gynecol Oncol 2017; 28: e48. doi: 10.3802/jgo.2017.28. $\mathrm{e} 48$

30. Cress RD, Chen YS, Morris CR, Petersen M, Leiserowitz GS. Characteristics of long-term survivors of epithelial ovarian cancer. Obstet Gynecol 2015; 126: 491-7. doi: 10.1097/AOG.0000000000000981

31. Brown J, Frumovitz M. Mucinous tumors of the ovary: current thoughts on diagnosis and management. Curr Oncol Rep 2014; 16: 389. doi: 10.1007/ s11912-014-0389-x

32. Marchetti C, Kristeleit R, McCormack M, Mould T, Olaitan A, Widschwendter $M$, et al. Outcome of patients with advanced ovarian cancer who do not undergo debulking surgery: A single institution retrospective review. Gynecol Oncol 2017; 144: 57-60. doi: 10.1016/j.ygyno.2016.11.001

33. Gill SE, McGree ME, Weaver AL, Cliby WA, Langstraat CL. Optimizing the treatment of ovarian cancer: Neoadjuvant chemotherapy and interval debulking versus primary debulking surgery for epithelial ovarian cancers likely to have suboptimal resection. Gynecol Oncol 2017; 144: 266-73. doi: 10.1016/j.ygyno.2016.11.021 